\title{
QUALIDADE DE LUZ NO DESEMPENHO DA RÃ-TOURO NA FASE DE TERMINAÇÃO
}

\author{
CASTRO, Thiago Rodrigues ${ }^{1}$ \\ PEREIRA, Marcelo Maia ${ }^{2}$ \\ RIBEIRO FILHO, Oswaldo Pinto ${ }^{3}$ \\ NAVARRO, Rodrigo Diana ${ }^{4}$ \\ CAMARGO FILHO, Cláudio Barberini ${ }^{5}$
}

\begin{abstract}
RESUMO: Os fatores abióticos temperatura, umidade e fotoperíodo influenciam o desenvolvimento e o metabolismo dos anfíbios, a partir disto objetivou-se verificar a influência das luzes vermelha, azul e verde no desempenho da rã-touro na fase de terminação. Foram utilizadas 480 rãs-touro divididas em três tratamentos e quatro repetições. Os parâmetros avaliados foram peso vivo do animal, do corpo adiposo, da carcaça e da pele, ganho de peso por dia, o índice lipossomático e os rendimentos de carcaça e pele. Os resultados encontrados para os parâmetros avaliados não diferenciaram $(\mathrm{p}>0,05)$ para as diferentes cores de luz. As luzes de cor vermelha, azul e verde não influenciaram o desempenho da rã-touro na fase de terminação, mais estudos devem ser realizados sobre outros aspectos relacionados à criação desta espécie.
\end{abstract}

Palavras-chave: Lithobates catesbeianus. Luz monocromática. Pós-metamorfose. Ranicultura.

\section{LIGHT QUALITY ON THE PERFORMANCE OF THE BULLFROG IN THE FINISHING PHASE}

SUMMARY: The abiotic factors of temperature, humidity and photoperiod influence the development and metabolism of amphibians, from this we aimed was verifying the influence of red, blue and green lights on the performance of the bullfrog in the finishing phase. We used 480 bullfrogs divided into three treatments and four replications. The parameters were live animal weight, body fatty, carcass and skin, weight gain per day, the fatty somatic index, the carcass and skin yield. The results for the evaluated parameters did not differ ( $>>0.05)$ for the different colors of light. The lights of red, blue and green did not affect the performance of the bullfrog in the finishing phase, more studies should be conducted on other aspects related to the creation of this specie.

Keywords: Lithobates catesbeianus. Monochromatic light. Post-metamorphosis. Frog culture.

\section{INTRODUÇÃO}

Os fatores abióticos que influenciam o desenvolvimento e o metabolismo dos anfíbios anuros são o fotoperíodo (CAMARGO et al., 1999; FIGUEIREDO et al., 2001; BAMBOZZI et al., 2004; FILADELFI et al., 2005), a temperatura (FIGUEIREDO et al., 1999; BRAGA; LIMA, 2001; SILVA et al., 2002; PETERSEN; GLEESON, 2009) e umidade (Cui et al., 2011).

Além do período de exposição à luz influenciar os anfíbios, ondas curtas de iluminação estimularam o crescimento em peso, comprimento e metamorfose de girinos de Rana temporária (RUCHIN, 2003).

\footnotetext{
${ }^{1}$ Zootecnista, Universidade Federal de Lavras, CEP 37200-000, Lavras, MG. thiagorcz@ yahoo.com.br ${ }^{2}$ Engenheiro Agrônomo, M.Sc., Universidade Estadual Paulista, Centro de Aquicultura, Via de Acesso Prof. Paulo Donato Castellane, s/n, CEP 14.884-900, Jaboticabal, SP. autor para correspondência. mmaiap2001@yahoo.com.br ${ }^{3}$ Biólogo, Universidade Federal de Viçosa, Av. Ph. Rolfs, s/n, CEP 36570-000, Viçosa, MG. oribeiro@ufv.br ${ }^{4}$ Zootecnista, Universidade de Brasília, FAV, Caixa Postal 4.508 - CEP 70.910-970, Brasília, DF. navarrounb@gmail.com

${ }^{5}$ Zootecnista, Instituto Federal do Espírito Santo, Departamento de Aquicultura, CEP 29520-000 Alegre, ES. cbcfilho@ifes.edu.br
} 
Em peixes, a cor da iluminação afetou a resposta ao estresse em jundiá (Rhamdia quelen) (BARCELLOS et al., 2006), a luz de cor azul melhorou o bem estar da tilápia do Nilo (VOLPATO; BARRETO, 2001) e com iluminação azul a reprodução da tilápia do nilo foi mais frequente e intensas (VOLPATO et al, 2004).

A luz interferiu nas atividades de Bufo marinus, Bufo typhonius, Dendobrates auratus, Leptodactylus pentadactylus e Physalaemus pustulosus, revelando possuir importante papel na ecologia de anfíbios anuros (JAEGER; HAILMAN, 1981).

Para o animal em confinamento é importante oferecer as melhores condições de criação e de bem estar, para que ele apresente a resposta desejável em menor tempo possível, com maior rentabilidade e melhor qualidade de carne.

O trabalho teve como objetivo verificar a influência das luzes vermelha, azul e verde no desempenho da rã-touro na fase de terminação, por meio da avaliação dos parâmetros de desempenho.

\section{MATERIAL E MÉTODOS}

O experimento foi conduzido no Ranário Experimental da Universidade Federal de Viçosa, durante 30 dias.

Os tratamentos foram utilização de luz monocromática, com lâmpada de $30 \mathrm{~W}$ de coloração vermelha $(625-740 \mathrm{~nm})$, azul $(440-485 \mathrm{~nm})$ e verde $(500-565 \mathrm{~nm})$, para iluminar as baias durante o período de 12 horas de luz que foi controlado por um timer, sendo a intensidade luminosa, de 500 Lux, medida por um luxímetro.

O delineamento foi inteiramente casualizado com três tratamentos (luz vermelha, azul e verde) e quatro repetições (cada repetição foi o lote de animais alojados dentro da baia).

Foram utilizadas baias de 1,54 m2 de área, um metro de altura, uma cobertura fixa de lona plástica para impedir entrada de luz do ambiente exterior, uma lâmpada, com uma parte seca que tem dois cochos e uma canaleta com água.

Em cada baia um lote com quarenta rãs-touro (Lithobates catesbeainus) foi alojado, as quais foram pesadas com uma balança de $0,01 \mathrm{~g}$ de precisão e apresentaram peso médio de $120,75 \pm 2,75 \mathrm{~g}$.

As rãs foram alimentadas com ração comercial contendo $42 \%$ de proteína bruta (Tabela 1) e foi fornecida duas vezes ao dia (7 horas e às 13 horas) na quantidade de 2,5\% do peso vivo dos animais (LIMA et al., 2003), valor corrigido a cada 10 dias com a pesagem de todos os animais, mais $20 \mathrm{~g}$ de larva de mosca doméstica. Diariamente a água da canaleta foi renovada em sua totalidade, a temperatura foi medida através de um termômetro de máximo e mínimo e verificado a presença de animais mortos.

Tabela 1. Composição centesimal da ração comercial utilizadas na alimentação da rã-touro (Lithobates catesbeianus)

(Continua)

\begin{tabular}{ll}
\hline & Níveis de garantia (\%) \\
\hline Cálcio (máximo) & 4,5 \\
Extrato etéreo (mínimo) & 9,0 \\
Fósforo (mínimo) & 2,0 \\
Matéria fibrosa (máximo) & 4,0
\end{tabular}


Tabela 1. Composição centesimal da ração comercial utilizadas na alimentação da rã-touro (Lithobates catesbeianus)

(Conclusão)

Níveis de garantia (\%)

$\begin{array}{ll}\text { Matéria mineral (máximo) } & 15,0 \\ \text { Proteína bruta (mínimo) } & 42,0 \\ \text { Umidade (máximo) } & 12,0\end{array}$

Composição básica: farelo de soja, farinha de peixe, farelo de trigo, farelo de glúten de milho $60 \%$, farinha de carne e ossos, milho, farinha de sangue, óleo de peixe, carbonato de cálcio, fosfato bicálcico, sal, suplemento vitamínico ${ }^{1}$, mineral $^{2}$ e antioxidante (etoxiquina $-250 \mathrm{mg}$ ).

${ }^{1}$ Composição por $\mathrm{Kg}$ : vitamina $\mathrm{A}-12.000$ UI,vitamina $\mathrm{D}_{3}-4.000 \mathrm{UI}$, vitamina $\mathrm{E}-150 \mathrm{UI}$, vitamina $\mathrm{K}-10 \mathrm{UI}$, ácido fólico $-10 \mathrm{mg}$, biotina $-0,8 \mathrm{mg}$, colina $-500 \mathrm{~g}$, niacina $-150 \mathrm{~g}$, pantotenato de cálcio $-50 \mathrm{mg}$, tiamina -30

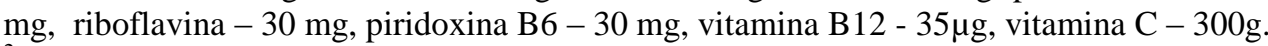

${ }^{2}$ Composição por $\mathrm{Kg}$ : Mg - 700mg, Mn- 30 mg, Zn - 200 mg, Cu - 15 mg, Fe - 100 mg, I - 1 mg, Se - 0,3 mg.

Após 30 dias de exposição aos tratamentos, 10 rãs de cada baia foram pesadas, insensibilizadas por termonarcose e posteriormente sacrificadas, segundo Figueiredo et al. (2001), onde o corpo adiposo, a carcaça e a pele foram coletadas e pesadas em balança com $0,01 \mathrm{~g}$ de precisão.

Os parâmetros avaliados foram peso vivo das rãs (g), do corpo adiposo $(\mathrm{g})$, da carcaça (g) e da pele (g), ganho de peso diário (g/dia), o índice lipossomático (ILS) e os rendimentos de carcaça (RC) e pele (RP).

Para calcular o índice lipossomático foi utilizada a fórmula: ILS (\%) = PCA/PV x 100, onde $\mathrm{PCA}=$ peso do corpo adiposo $(\mathrm{g})$. Para cálculo do rendimento de carcaça foi utilizada a fórmula: $\mathrm{RC}(\%)=$ PC/PV x 100, onde PC = peso da carcaça $(\mathrm{g})$ e para calcular o rendimento de pele foi utilizada a fórmula: $\mathrm{RP}(\%)=\mathrm{PP} / \mathrm{PV} \times 100$, onde $\mathrm{PP}=$ peso da pele $(\mathrm{g})$.

As análises de variância (ANOVA) e o teste de Sdudent-Newman-Keuls a $5 \%$ de probabilidade foram realizados com auxílio do programa SAEG (2007).

\section{RESULTADOS E DISCUSSÃO}

A temperatura máxima e mínima das baias foi de $29^{\circ} \mathrm{C} \pm 2$ e $24^{\circ} \mathrm{C} \pm 1$.

Os anfíbios são animais heterotérmicos, e a temperatura encontrada estão dentro da faixa considerada ótima para a criação de rã de 25,1 a 30,4 ${ }^{\circ} \mathrm{C}$ (FIGUEIREDO et al., 1999; BRAGA; LIMA, 2001) e para os processos fisiológicos dos anfíbios (SILVA et al., 2002; PETERSEN; GLEESON, 2009).

A mortalidade dos animais submetidos à luz vermelha foi de 3,33\%, à luz azul foi de $4,10 \%$ e à luz verde foi de 3,33\%, foi inferior aos $13,9 \%$ para rã-touro na fase de engorda em ranário comercial (LIMA et al., 2003), mortalidades altas em ranários comerciais estão associados a manejos inadequados (HIPOLITO et al., 2004).

Os resultados encontrados para os parâmetros peso vivo final, do corpo adiposo, da pele, da carcaça, índice lipossomático, rendimento de pele, rendimento de carcaça e ganho de peso não apresentaram diferenças estatísticas (p>0,05) para as diferentes cores de luz (Tabela 2), ou seja, as luzes adotadas não influenciaram o desempenho da rã-touro, resultado este diferente para girinos de Rana temporaria onde a luz azul obteve pior desempenho (RUCHIN, 2003), indicando que a qualidade da luz pode influenciar o desempenho somente durante a fase larvária ou de girino. 
Tabela 2. Peso vivo inicial (PI) e final (PF), do corpo adiposo (PCA), da pele (PP), da carcaça (PC), índice lipossomático (ILS), rendimento de pele (RP), rendimento de carcaça (RC) e ganho de peso (GP) de rã-touro (Lithobates catesbeianus) submetida a diferentes cores de luz

\begin{tabular}{lrrrr}
\hline Parâmetros & \multicolumn{3}{c}{ Cores } & CV (\%) \\
\cline { 2 - 4 } & Vermelho & \multicolumn{1}{c}{ Azul } & Verde & \\
\cline { 2 - 4 } PI (g) & $120,50^{\mathrm{a}}$ & $121,30^{\mathrm{a}}$ & $120,80^{\mathrm{a}}$ & - \\
PF (g) & $223,14 \pm 22,10^{\mathrm{a}}$ & $215,93 \pm 23,12^{\mathrm{a}}$ & $214,90 \pm 23,12^{\mathrm{a}}$ & 10,64 \\
GPD (g/dia) & $3,42 \pm 0,74^{\mathrm{a}}$ & $3,15 \pm 0,77^{\mathrm{a}}$ & $3,13 \pm 0,81^{\mathrm{a}}$ & 23,88 \\
PCA (g) & $17,47 \pm 3,78^{\mathrm{a}}$ & $17,63 \pm 3,45^{\mathrm{a}}$ & $15,79 \pm 3,89^{\mathrm{a}}$ & 21,90 \\
PP (g) & $22,38 \pm 3,00^{\mathrm{a}}$ & $21,75 \pm 3,50^{\mathrm{a}}$ & $21,16 \pm 3,21^{\mathrm{a}}$ & 14,92 \\
PC (g) & $111,91 \pm 14,31^{\mathrm{a}}$ & $108,62 \pm 13,56^{\mathrm{a}}$ & $109,32 \pm 13,87^{\mathrm{a}}$ & 12,65 \\
ILS (\%) & $7,83 \pm 1,46^{\mathrm{a}}$ & $8,12 \pm 1,02^{\mathrm{a}}$ & $7,29 \pm 1,34^{\mathrm{a}}$ & 16,64 \\
RP (\%) & $10,03 \pm 0,95^{\mathrm{a}}$ & $10,06 \pm 1,09^{\mathrm{a}}$ & $9,83 \pm 0,82^{\mathrm{a}}$ & 9,69 \\
RC (\%) & $50,15 \pm 3,95^{\mathrm{a}}$ & $50,31 \pm 3,36^{\mathrm{a}}$ & $50,89 \pm 3,28^{\mathrm{a}}$ & 7,03 \\
\hline
\end{tabular}

*Médias ( \pm Erro padrão) na mesma linha seguidas da mesma letra não diferem estatisticamente ao nível de 5\%.

O peso médio final dos animais de 223,14 g (luz vermelha), 215,93 g (luz azul) e 214,90 g (luz verde) dos três tratamentos foram superior a $200 \mathrm{~g}$, peso considerado mínimo para abate comercial (LIMA; AGOSTINHO, 1992).

$\mathrm{O}$ ganho de peso de 3,42 g/dia foi superior aos 2,80 g/dia para rã-touro na fase de engorda em ranário comercial sem controle de iluminação (LIMA et al., 2003), mas as condições deste trabalho foram mais controladas e com isso permitiu melhor desempenho.

O índice lipossomático encontrado de $8,12 \%$ foi superior aos 5,51\% para rã-touro submetida a 12 horas de luz branca e a temperatura constante de 29 oC (Figueiredo et al., 2001). O corpo adiposo da rãtouro exerce função de armazenamento de gordura para ser utilizado pelo animal durante o período de reprodução e como reserva durante as baixas temperaturas (NAVARRO et al., 2005; PEREIRA et al., 2011), mas ainda não se tem um valor definido como ideal para o índice lipossomático.

O rendimento de carcaça encontrado de 50,89\% foi inferior aos 58,8\% para rã-touro submetida a 12 horas de luz branca (FIGUEIREDO et al., 1999), esses diferentes valores podem ser atribuídos a distinta qualidade entre as rações adotadas nos trabalhos acima citados (CASALI et al., 2005).

O rendimento de pele encontrado de $10,06 \%$ foi semelhante aos $10,02 \%$ para rã-touro criadas em mini baias (CASALI et al., 2005), a pele é um subproduto que pode ser aproveitamento pela indústria para fabricação de cintos, bolsas, luvas, sapatos e na medicina para tratamentos de queimaduras (SILVA; OLIVEIRA, 1994).

Os resultados encontrados para alguns parâmetros neste trabalho diferiram dos encontrados na literatura para rã-touro, podendo levantar a hipótese de diferença entre o desempenho para esta espécie criada sob a luz branca e as luzes adotadas neste trabalho e que devem ser futuramente investigadas, além de estudos sobre aspectos reprodutivos e metabólicos relacionados ao estresse.

\section{CONCLUSÃO}

As luzes de cor vermelha, azul e verde não influenciaram o desempenho da rã-touro na fase de terminação. 


\section{REFERÊNCIAS}

BAMBOZZI, A.C. et al. Efeito do fotoperíodo sobre o desenvolvimento de girinos de rã-touro (Rana catesbeiana Shaw, 1802). Revista Brasileira de Zootecnia, v.3, p. 1-7, 2004.

BARCELLOS, L.J.G. et al. The color of ilumination affects the stress response of jundia (Rhamdia quelen, Quoy \& Gaimard, Heptapteridae). Ciência Rural, v.36, p. 1249-1252, 2006.

BRAGA, L.G.T.; LIMA, S.L. Influência da temperatura ambiente no desempenho da rã-touro, Rana catesbeiana (Shaw, 1802), na fase de recria. Revista Brasileira de Zootecnia, v.30, p. 1659-1663, 2001.

CAMARGO, C.R.; VISCONTI, M.A.; CASTRUCCI, A.M.L. Physiological color change in the Bullfrog, Rana catesbeiana. Journal of Experiemntal Zoology, v.283, p. 160-169, 1999.

CASALI, A.P.; MOURA, O.M.; LIMA, S.L. Rações comerciais e o rendimento de carcaça e subprodutos de rã-touro. Ciência Rural, v.35, p. 1172-1178, 2005.

CUI , J.G. et al. Circadian rhythm of calling behavior in the emei music frog (Babina dauchina) is associated with habitat and frog relative humidity. Asian Herpetological Research, v.2, p. 149-154, 2011.

FIGUEIREDO, M.R.C. et al. Efeito da temperatura e do fotoperíodo sobre o desenvolvimento do aparelho reprodutor de rã-touro (Rana catesbeiana Shaw, 1802). Revista Brasileira de Zootecnia, v. 30, p. 916923, 2001.

FIGUEIREDO, M.R.C. et al. Efeito da temperatura sobre o desempenho da rã-touro (Rana catesbeiana Shaw, 1802). Revista Brasileira de Zootecnia, v. 28, p. 661-667, 1999.

FILADELFI, A.M.C.; VIEIRA, A.; LOUZADA, F.M. Circadian rhythm of physiological color change in the amphibian Bufo ictericus under different photoperiods. Comparative Biochemistry and Physiology, Part A, v. 142, p. 370-375, 2005.

HIPOLITO, M. et al. Observações de lesões post-mortem em rãs-touro (Rana catesbeiana) Sham, 1802) abatidas comercialmente no estado de São Paulo, Brasil. Arquivos Instituto Biológico, v. 71, p. 237-241, 2004.

JAEGER, R.G.; HAILMAN, J.P. Activity of neotropical frogs in relation to ambient light. Biotropica, v. 13, p. 59-65, 1981.

LIMA, S.L.; AGOSTINHO, C.A. A tecnologia de criação de rãs. Viçosa: UFV. 168 p. 1992.

LIMA, S.L.; CASALI, A.P.; AGOSTINHO, C.A. Desempenho zootécnico e percentual de consumo de alimento de rã-touro (Rana catesbeiana) na fase de recria do sistema anfigranja. Revista Brasileira de Zootecnia, v.31, p. 505-511, 2003.

NAVARRO, R.D. et al. Efeito do hormônio 17- $\alpha$-metil-testosterona nos índices somáticos de Rana catesbeiana. Zootecnia Tropical, v. 23, p. 319-325, 2005.

PEREIRA, M.M. et al. Tecido hepático e corpo adiposo de rã-touro (lithobates catesbeianus) submetido a diferentes fotoperíodos. Acta Veterinaria Brasilica, v. 5, p. 76-80, 2011.

PETERSEN, A.M.; GLEESON, T.T. Skeletal muscle substrate utilization is altered by acute and acclamatory temperature in the American bullfrog (Lithobates catesbeiana). The Journal of Experimental Biology, v. 212, p. 2378-2385, 2009. 
RUCHIN, A.B. Effect of monochromatic light on the growth and development of brow frog (Rana temporaria) larvae. Biology Bulletin, v. 30, p. 482-484, 2003.

SAEG. Sistema para Análises Estatísticas, Versão 9.1: Fundação Arthur Bernardes - UFV. Viçosa, 2007.

SILVA, A.S.; NUNES, W.M.S.; SILVA, C.S. Interferência sazonal no metabolismo da Rana catesbeiana Shaw, 1802 (Anura, Ranidae). Revista Brasileira de Zoociências, v.4, p. 45-56, 2002.

SILVA, N.R.; OLIVEIRA, L.A. Ocorrência de Salmonella na carne de rã (Rana catesbeiana, Shaw 1803). Higiene Alimentar, v.8, p. 36-40, 1994.

VOLPATO, G.L.; BARRETO, R.E. Environmental blue light prevents stress in the fish nile tilapia. Brazilian Journal of Medical and Biological Research, v.34, p. 1041-1045, 2001.

VOLPATO, G.L.; DUARTE, C.R.A.; LUCHIARI, A.C. Environmental color affects nile tilapia reproduction. Brazilian Journal of Medical and Biological Research, v.37, p. 479-483, 2004. 